\title{
THE INTERNAL PROPER MOTIONS OF STARS IN THE OPEN CLUSTER M35
}

\author{
Bernard J. McNamara ${ }^{1}$, Thomas E. Harrison ${ }^{1}$, Barbara E. McArthur ${ }^{2}$, and G. Fritz Benedict ${ }^{2}$ \\ ${ }^{1}$ Department of Astronomy, New Mexico State University, Box 30001, MSC 4500, Las Cruces, NM 88003-8001, USA \\ 2 McDonald Observatory, University of Texas, Austin, TX 78712, USA \\ Received 2011 May 13; accepted 2011 June 9; published 2011 July 12
}

\begin{abstract}
Relative proper motions, based on 108 orbits of Hubble Space Telescope Fine Guidance Sensor data extending from 1992 to 2006, are reported for 74 stars in the open cluster M35 (NGC 2168). A subset of 22 of these objects are then used to compute the cluster's internal proper motion dispersions in both right ascension and declination. We find that these dispersions are equal to within their measurement errors. The average one-dimensional dispersion is $0.018 \pm 0.002$ arcsec century $^{-1}$. When combined with the M35 radial velocity dispersion of $0.65 \pm 0.10 \mathrm{~km} \mathrm{~s}^{-1}$ found by Geller et al., this produces a cluster distance of $762 \pm 145 \mathrm{pc}$. Using isochrone fits to the cluster main sequence, this distance suggests that M35 has an age of about 133 Myr. Although this age is consistent with that typically found for M35, the formal error in the dynamical distance of $\pm 19 \%$ can accommodate ages between $65 \mathrm{Myr}$ and $201 \mathrm{Myr}$.
\end{abstract}

Key words: open clusters and associations: individual (M35) - proper motions - stars: kinematics and dynamics

\section{INTRODUCTION}

Measurements of the internal proper motions within star clusters are important for several reasons. These measurements provide constraints to models of the dynamical evolution of these objects. Second, when combined with radial velocity data they allow a distance to be determined in a manner that is independent of star formation theories, estimates of the cluster's reddening, or metallicity. Third, they allow us to compare masses determined by application of the virial theorem to cluster masses estimated by direct star counts. To exploit these advantages internal proper motions have been measured for a number of clusters: M45 (Jones 1970; Geffert et al. 1995), the Orion Nebulae Cluster (McNamara 1976), M15 (Cudworth 1976a; McNamara et al. 2004), M3 (Cudworth 1979a), M5 (Cudworth 1979b), M92 (Cudworth 1976b), M11 (McNamara \& Sanders 1977), NGC 6494 (McNamara \& Sanders 1983), and $\omega$ Centauri (van der Marel \& Anderson 2010). However, M35 is a particularly interesting object to study because it provides information about the dynamical state of an intermediate-age cluster. In addition, over a decade of precise Hubble Space Telescope/Fine Guidance Sensor (HST/ FGS) M35 positional measurements are available and new data continue to be collected on a yearly basis.

M35 (NGC 2168) is a prominent galactic star cluster with a well-populated main sequence down to stars with masses of at least $0.2 M_{\odot}$. Its distance has been estimated by several investigators with values ranging from 731 to 912 pc depending on which combination of cluster reddening, age, and metallicity were employed in these studies. A convenient list of M35 distances can be found in Kalirai et al. (2003). $E(B-V)$ values range from 0.17 to $0.22 \mathrm{mag}$, ages from 85 to $200 \mathrm{Myr}$, and $[\mathrm{Fe} / \mathrm{H}]$ values from -0.18 to -0.21 . Its stellar membership, based on relative proper motions, has been previously determined to a limiting apparent visual magnitude of about 15 by Ebbighausen (1942), Cudworth (1971), and McNamara \& Sekiguchi (1986a). The latter investigators also provided the first estimate of M35's internal proper motion dispersion, $0.022 \pm 0.003$ arcsec century $^{-1}$ (McNamara \& Sekiguchi 1986b). Leonard \& Merritt (1989) used dynamical models to measure the mass within the cluster's central $3.75 \mathrm{pc}$. At the $95 \%$ confidence level, their analysis found a value of $2400 \pm$ $800 M_{\odot}$.

In the next section, we describe the FGS data used in this study. We then describe the method used to derive the relative proper motions and internal proper motion dispersions. Section 5 provides estimates of the cluster's distance and age. Results are summarized in the last section.

\section{DATA}

Positional $x, y$ measurements for 100 stars in M35 were obtained from 108 HST/FGS orbits extending from 1992 to 2006. The FGS is a nulling interferometer that is used to obtain precise stellar positions. Three of these instruments are carried by the HST in its radial bays and each FGS views a bent rectangle region of the sky whose shape is that of a "pickle." A pickle covers about $67 \operatorname{arcmin}^{2}$ of sky with a maximum separation along the long axis of about 18.6 arcmin. Positional $x, y$ measurements are obtained by the FGS using the position (POS) mode. This mode is employed when individual stellar positions are desired, for instance, as part of a parallax study. A detailed discussion of the way in which $x, y$ values are obtained in POS mode has been provided in Hemenway et al. (1997), so only a brief overview is given here. In this mode only one star is observed at a time. The accuracy of an individual measurement is about 2 mas for stars having $3.0<V<16.8$ (Benedict et al. 1992). Approximately 25 M35 stars were observed in each of the 108 data sets used in this study. Typically two of these stars are measured multiple times during an orbit to monitor the stability of the measured $x, y$ values. Although the number of M35 stars observed each orbit is about the same, different stars are observed throughout the year due to the changing FGS field of view with respect to M35.

A histogram of the number of measurements obtained for each of the 100 stars in our data set is shown in Figure 1(a). Figure 1(b) shows the distribution of epoch differences among these stars. Nineteen were measured less than four times and were dropped from our sample. Seven other stars with few measurements were also later dropped because inadequate data were available to compute their proper motions. As these figures 

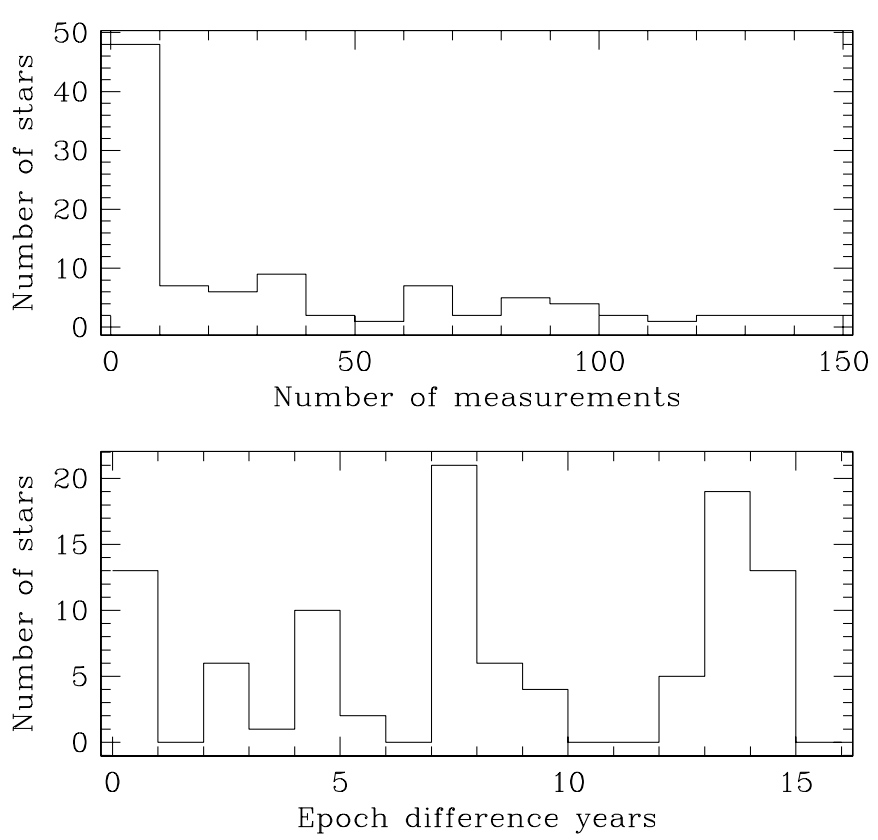

Figure 1. Top panel shows the distribution of the number of measurements available for the M35 stars in the HST FGS data base. The bottom panel shows the histogram of the epoch differences for these stars. Every few years several orbits of HST time are devoted to FGS measurements of M35 as part of this instrument's calibration process. These figures illustrate the highly non-uniform nature of the M35 data base.

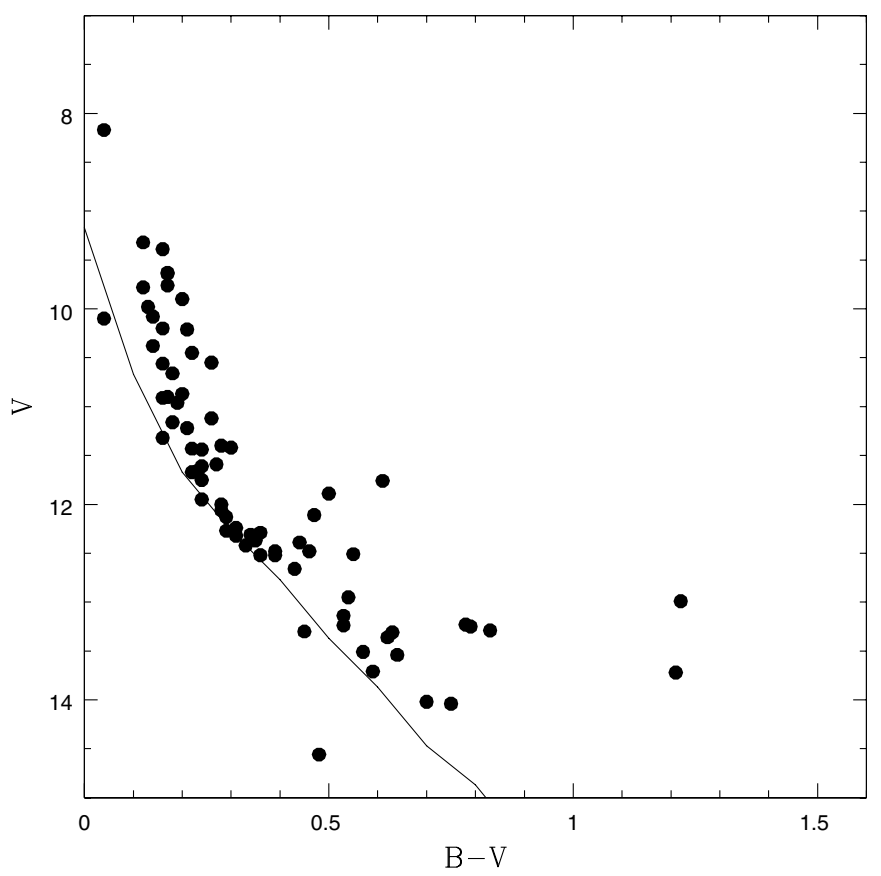

Figure 2. M35 color-magnitude diagram for 73 of the 74 stars for which proper motions will be determined. This figure indicates that several of these stars are likely to be field stars based on their location in this figure. The solid line is the zero-age main sequence taken from Allen (1976), reddened by $E(B-V)=$ 0.20 , and scaled to a distance of 805 pc (Geller et al. 2010).

show, the remaining data set is highly non-uniform both in the number of observations per star and in the epoch differences. This will result in proper motions that have unequal precisions. The maximum number of measurements obtained for an M35 star was 133 and the maximum time spanned by the observations was 14.03 years. Figure 2 shows a color-magnitude diagram of the remaining 74 stars in the sample (one star lacked a $V$

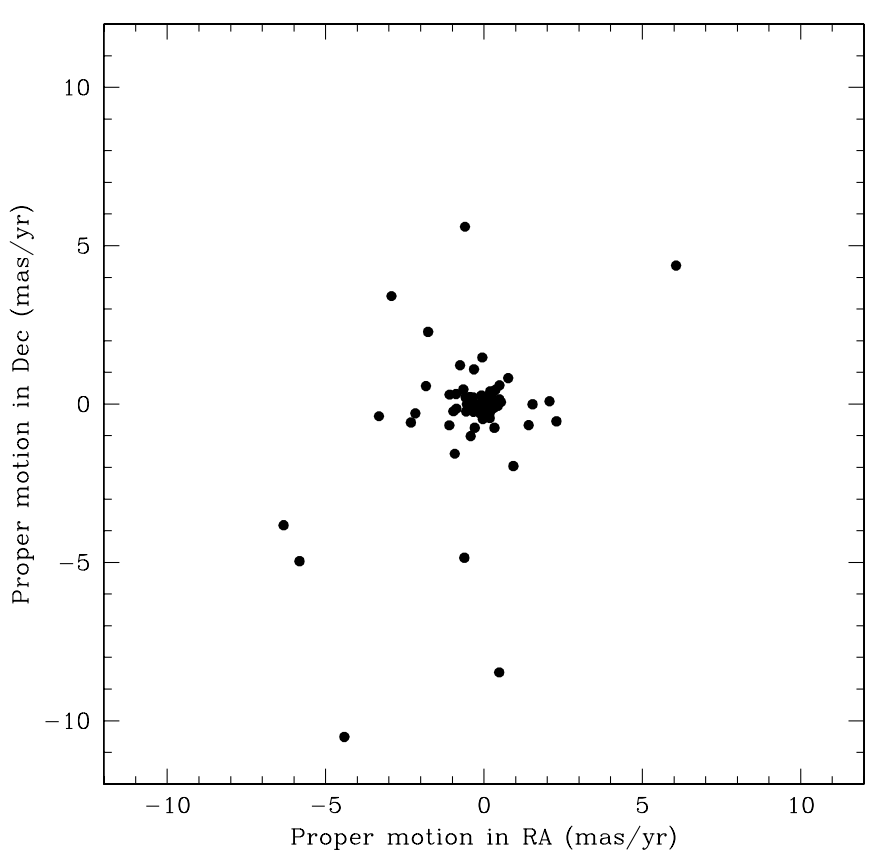

Figure 3. Distribution of M35 proper motions shows a well-defined concentration at zero proper motion. This reflects the fact that the proper motions were computed using cluster stars as reference objects. Stars located outside this central region are field stars. Several stars measured by the FGS in the region of M35 are field stars.

and $B-V$ value). Colors and magnitudes in this table were taken from Sung \& Bessell (1999). The solid line in this figure represents the zero-age main sequence (Allen 1976). It has been reddened by $E(B-V)=0.20$ and scaled to a distance of $805 \mathrm{pc}$ (Geller et al. 2010).

\section{DERIVATION OF THE PROPER MOTIONS}

The proper motions in this study were derived using M35 stars as reference objects. Individual data sets were rotated to align their $x, y$ coordinate system with right ascension and declination. One FGS data file was then adopted as a standard and all others were shifted in $x$ and $y$ to match the center of that frame. The choice of this standard file is arbitrary. The proper motions were then determined by fitting the change in position of a star to straight lines in both right ascension and declination. The slopes of these lines represent the proper motion. This procedure is essentially the same as the commonly used plate overlap technique.

To avoid distortions that might exist over the large field of view of the FGS, localized reference frames consisting of stars within 300 arcsec of the target star were used. Typically these frames contained about a dozen stars. Occasionally a field star whose motion was subtracted in the solution was used to augment the number of reference stars. The use of higher order terms was explored in the proper motion solution, but only linear terms were found to be necessary. The vector-point diagram of all stars measured in this study is shown in Figure 3. Table 1 provides a list of M35 proper motions. Column 1 contains the star number that is used in the FGS data files. If the number is followed by an asterisk that star is used in the computation of the internal proper motion dispersion. The second column provides the star number in McNamara \& Sekiguchi (1986a). Column 3 contains the number of measurements that were available for each star; Column 4 provides the epoch difference in units of years. The following columns provide the proper motions 
Table 1

M35 Proper Motions

\begin{tabular}{|c|c|c|c|c|c|c|c|}
\hline$\overline{H S T}$ & $\mathrm{McN}$ & Measurements & Epoch Diff. & $\begin{array}{c}\mu_{r a} \pm \text { error } \\
\left(\operatorname{arcsec} \text { century }^{-1}\right)\end{array}$ & $\left.\begin{array}{c}\mu_{\mathrm{dec}} \pm \text { error } \\
(\operatorname{arcsec} \text { century } \\
-1\end{array}\right)$ & V & $\overline{B-V}$ \\
\hline 9 & 34 & 5 & 4.28 & $-0.599 \pm 0.399$ & $5.600 \pm 0.941$ & 13.36 & 0.62 \\
\hline 419 & 67 & 5 & 4.28 & $1.411 \pm 0.729$ & $-0.668 \pm 0.763$ & 12.27 & 0.29 \\
\hline $470^{*}$ & 397 & 86 & 12.70 & $-0.444 \pm 0.062$ & $0.223 \pm 0.047$ & 12.42 & 0.33 \\
\hline 478 & 254 & 22 & 13.85 & $0.482 \pm 0.098$ & $-8.473 \pm 0.100$ & 13.25 & 0.79 \\
\hline 489 & 454 & 29 & 12.27 & $-5.823 \pm 0.164$ & $-4.962 \pm 0.079$ & 13.23 & 0.78 \\
\hline $496^{*}$ & 350 & 85 & 14.03 & $0.156 \pm 0.059$ & $0.181 \pm 0.037$ & 9.76 & 0.17 \\
\hline $509^{*}$ & 218 & 115 & 13.93 & $-0.109 \pm 0.050$ & $-0.196 \pm 0.037$ & 8.17 & 0.04 \\
\hline $514^{*}$ & 163 & 64 & 12.38 & $0.131 \pm 0.052$ & $0.057 \pm 0.059$ & 11.66 & 0.23 \\
\hline 539 & 77 & 19 & 8.06 & $0.023 \pm 0.148$ & $-0.414 \pm 0.208$ & 10.38 & 0.14 \\
\hline 554 & 257 & 10 & 7.95 & $0.300 \pm 0.168$ & $-0.127 \pm 0.165$ & 11.40 & 0.28 \\
\hline 584 & 64 & 5 & 7.95 & $-0.967 \pm 0.288$ & $-0.230 \pm 0.218$ & 11.95 & 0.24 \\
\hline 596 & 426 & 8 & 7.95 & $-0.343 \pm 0.159$ & $0.214 \pm 0.217$ & 11.59 & 0.27 \\
\hline 603 & 434 & 59 & 12.35 & $0.537 \pm 0.065$ & $0.062 \pm 0.053$ & 10.55 & 0.26 \\
\hline $607 *$ & 203 & 126 & 14.03 & $-0.051 \pm 0.037$ & $0.102 \pm 0.045$ & 10.90 & 0.17 \\
\hline 627 & 59 & 4 & 7.95 & $-3.317 \pm 0.489$ & $-0.385 \pm 0.459$ & 10.10 & 0.04 \\
\hline 628 & 60 & 5 & 7.95 & $6.066 \pm 0.446$ & $4.374 \pm 0.651$ & 11.76 & 0.61 \\
\hline 629 & 73 & 12 & 8.28 & $2.068 \pm 0.340$ & $0.086 \pm 0.164$ & 10.87 & 0.20 \\
\hline 631 & 364 & 69 & 7.96 & $0.482 \pm 0.090$ & $0.152 \pm 0.097$ & 10.66 & 0.18 \\
\hline $656^{*}$ & 405 & 70 & 13.93 & $-0.106 \pm 0.060$ & $-0.121 \pm 0.061$ & 11.61 & 0.24 \\
\hline 659 & 481 & 9 & 4.28 & $-6.335 \pm 1.020$ & $-32.124 \pm 0.675$ & 13.29 & 0.83 \\
\hline 674 & 338 & 6 & 7.95 & $0.093 \pm 0.230$ & $-0.182 \pm 0.330$ & 9.39 & 0.16 \\
\hline 679 & 375 & 13 & 7.95 & $0.181 \pm 0.104$ & $-0.444 \pm 0.218$ & 11.44 & 0.24 \\
\hline $688^{*}$ & 243 & 61 & 14.03 & $0.304 \pm 0.070$ & $0.034 \pm 0.060$ & 10.08 & 0.14 \\
\hline 699 & 394 & 4 & 13.93 & $-4.408 \pm 0.282$ & $-10.510 \pm 0.133$ & 12.51 & 0.55 \\
\hline 710 & 298 & 33 & 13.78 & $-0.540 \pm 0.091$ & $-0.006 \pm 0.068$ & 13.24 & 0.53 \\
\hline 712 & 98 & 29 & 7.95 & $-1.093 \pm 0.199$ & $-0.671 \pm 0.189$ & 12.48 & 0.46 \\
\hline 714 & 99 & 21 & 8.38 & $0.435 \pm 0.116$ & $-0.059 \pm 0.156$ & 12.11 & 0.47 \\
\hline 719 & 457 & 7 & 3.06 & $-0.755 \pm 0.537$ & $1.222 \pm 0.549$ & & \\
\hline 729 & 250 & 9 & 7.96 & $0.162 \pm 0.171$ & $0.007 \pm 0.271$ & 11.75 & 0.24 \\
\hline $732 *$ & 247 & 142 & 14.03 & $-0.126 \pm 0.043$ & $-0.144 \pm 0.038$ & 10.56 & 0.16 \\
\hline 737 & 251 & 39 & 9.03 & $0.232 \pm 0.114$ & $0.241 \pm 0.117$ & 12.32 & 0.31 \\
\hline 756 & 120 & 32 & 8.38 & $-0.652 \pm 0.168$ & $0.461 \pm 0.143$ & 9.64 & 0.17 \\
\hline 797 & 502 & 7 & 4.28 & $1.534 \pm 0.701$ & $-0.009 \pm 1.192$ & 9.78 & 0.12 \\
\hline 798 & 466 & 48 & 8.38 & $-0.306 \pm 0.092$ & $-0.022 \pm 0.086$ & 12.24 & 0.31 \\
\hline 817 & 188 & 24 & 13.85 & $-0.080 \pm 0.078$ & $0.271 \pm 0.073$ & 10.91 & 0.16 \\
\hline 845 & 240 & 129 & 14.03 & $-0.565 \pm 0.047$ & $-0.233 \pm 0.053$ & 11.67 & 0.22 \\
\hline $853^{*}$ & 277 & 144 & 14.03 & $0.062 \pm 0.040$ & $-0.225 \pm 0.034$ & 10.21 & 0.21 \\
\hline 856 & 61 & 4 & 4.28 & $0.928 \pm 0.238$ & $-1.960 \pm 0.330$ & 13.51 & 0.57 \\
\hline 862 & 449 & 37 & 13.93 & $-0.620 \pm 0.072$ & $-4.854 \pm 0.083$ & 12.99 & 1.22 \\
\hline $868^{*}$ & 297 & 92 & 14.03 & $0.228 \pm 0.053$ & $-0.069 \pm 0.039$ & 10.96 & 0.19 \\
\hline 875 & 288 & 9 & 2.89 & $-0.293 \pm 0.566$ & $-0.750 \pm 0.715$ & 12.37 & 0.35 \\
\hline 877 & 55 & 5 & 4.28 & $-2.921 \pm 0.934$ & $3.410 \pm 0.447$ & 12.06 & 0.28 \\
\hline 883 & 66 & 8 & 12.94 & $-0.581 \pm 0.053$ & $0.211 \pm 0.086$ & 12.48 & 0.39 \\
\hline 885 & 341 & 11 & 13.85 & $-1.764 \pm 0.303$ & $2.280 \pm 0.090$ & 13.72 & 1.21 \\
\hline $894 *$ & 134 & 65 & 13.93 & $0.128 \pm 0.045$ & $0.263 \pm 0.052$ & 10.20 & 0.16 \\
\hline 897 & 129 & 12 & 13.85 & $-0.420 \pm 0.107$ & $-1.013 \pm 0.090$ & 13.14 & 0.53 \\
\hline 906 & 53 & 7 & 4.28 & $-1.085 \pm 0.498$ & $0.297 \pm 0.579$ & 12.00 & 0.28 \\
\hline $912^{*}$ & 260 & 102 & 13.85 & $0.195 \pm 0.063$ & $-0.033 \pm 0.063$ & 12.31 & 0.34 \\
\hline 913 & & 32 & 9.03 & $0.329 \pm 0.106$ & $-0.751 \pm 0.129$ & 13.71 & 0.59 \\
\hline 915 & 456 & 40 & 9.03 & $-0.338 \pm 0.105$ & $-0.243 \pm 0.092$ & 13.54 & 0.64 \\
\hline $918^{*}$ & 316 & 86 & 14.03 & $-0.126 \pm 0.051$ & $0.002 \pm 0.059$ & 9.98 & 0.13 \\
\hline 919 & 179 & 7 & 7.95 & $0.368 \pm 0.222$ & $-0.009 \pm 0.263$ & 9.32 & 0.12 \\
\hline $932 *$ & 294 & 83 & 9.03 & $-0.135 \pm 0.079$ & $-0.291 \pm 0.080$ & 11.16 & 0.18 \\
\hline 935 & 428 & 75 & 14.00 & $0.426 \pm 0.077$ & $-0.023 \pm 0.075$ & 11.12 & 0.26 \\
\hline 946 & 315 & 7 & 7.95 & $-0.879 \pm 0.276$ & $0.319 \pm 0.403$ & 12.13 & 0.29 \\
\hline $947^{*}$ & 213 & 97 & 13.96 & $0.190 \pm 0.049$ & $-0.265 \pm 0.048$ & 11.22 & 0.21 \\
\hline 948 & 155 & 106 & 14.03 & $-6.329 \pm 0.053$ & $-3.828 \pm 0.044$ & 11.89 & 0.50 \\
\hline $950^{*}$ & 451 & 94 & 14.03 & $0.101 \pm 0.047$ & $-0.271 \pm 0.052$ & 11.32 & 0.16 \\
\hline $957^{*}$ & 401 & 133 & 14.03 & $0.001 \pm 0.037$ & $0.157 \pm 0.034$ & 9.63 & 0.17 \\
\hline 963 & 386 & 5 & 7.95 & $-0.053 \pm 0.400$ & $1.470 \pm 0.330$ & 12.39 & 0.44 \\
\hline 964 & 220 & 77 & 13.93 & $-0.871 \pm 0.063$ & $-0.145 \pm 0.048$ & 10.45 & 0.22 \\
\hline $967 *$ & 114 & 67 & 13.93 & $0.091 \pm 0.054$ & $-0.065 \pm 0.044$ & 9.90 & 0.20 \\
\hline 972 & 122 & 17 & 7.95 & $-2.307 \pm 0.404$ & $-0.583 \pm 0.386$ & 14.04 & 0.75 \\
\hline $975^{*}$ & 196 & 136 & 14.03 & $0.158 \pm 0.047$ & $0.192 \pm 0.042$ & 12.95 & 0.54 \\
\hline 978 & 171 & 44 & 13.93 & $-0.039 \pm 0.080$ & $-0.475 \pm 0.069$ & 12.52 & 0.39 \\
\hline
\end{tabular}


Table 1

(Continued)

\begin{tabular}{|c|c|c|c|c|c|c|c|}
\hline$H S T$ & $\mathrm{McN}$ & Measurements & Epoch Diff. & $\begin{array}{c}\mu_{r a} \pm \text { error } \\
\left(\operatorname{arcsec}^{2} \text { century }{ }^{-1}\right)\end{array}$ & $\begin{array}{c}\mu_{\mathrm{dec}} \pm \text { error } \\
\left(\operatorname{arcsec} \text { century }^{-1}\right)\end{array}$ & $V$ & $B-V$ \\
\hline $980 *$ & 361 & 93 & 13.79 & $0.242 \pm 0.067$ & $0.342 \pm 0.046$ & 11.42 & 0.30 \\
\hline $983^{*}$ & 396 & 86 & 13.93 & $0.041 \pm 0.054$ & $0.214 \pm 0.036$ & 11.43 & 0.22 \\
\hline 985 & 116 & 63 & 13.05 & $0.199 \pm 0.061$ & $0.406 \pm 0.063$ & 12.29 & 0.36 \\
\hline 989 & 412 & 38 & 13.92 & $-2.166 \pm 0.094$ & $-0.292 \pm 0.136$ & 13.30 & 0.45 \\
\hline 1001 & & 26 & 4.65 & $-1.834 \pm 0.280$ & $0.568 \pm 0.231$ & 14.56 & 0.48 \\
\hline 1002 & & 32 & 5.91 & $-0.145 \pm 0.202$ & $-0.190 \pm 0.249$ & 14.02 & 0.70 \\
\hline 1004 & & 35 & 5.98 & $-0.085 \pm 0.232$ & $-0.058 \pm 0.220$ & 13.31 & 0.63 \\
\hline 1006 & & 5 & 7.95 & $0.491 \pm 0.082$ & $0.591 \pm 0.338$ & 12.66 & 0.43 \\
\hline 1008 & & 11 & 2.06 & $-0.921 \pm 0.901$ & $-1.569 \pm 0.856$ & 12.520 & 0.36 \\
\hline
\end{tabular}

in right ascension and declination and their errors in units of arcseconds century ${ }^{-1}$. The last two columns provide the star's $V$ magnitude and $B-V$ color. As noted earlier, these values were taken from the work of Sung \& Bessell (1999). The central concentration at zero motion consists of cluster members; those with larger motions are likely to be field stars. The inclusion of stars that are not cluster members was expected based on the peculiar positions of several stars in Figure 2.

\section{COMPUTATION OF THE M35 INTERNAL PROPER MOTIONS}

The procedure used to compute the cluster's internal motion follows that discussed by Jones (1970). The observed proper motion dispersion in one coordinate, $\sigma_{o}$, is

$$
\sigma_{o}^{2}=\frac{1}{n-1} \sum_{k=1}^{n} \mu_{k}^{2}
$$

where $\mu_{k}$ is the proper motion of star $k$ and $n$ is the number of stars in the sample. The mean proper motion of the sample is assumed to be zero. The internal proper motion dispersion is then given by the equation

$$
\sigma_{i}^{2}=\sigma_{o}^{2}-\frac{1}{n} \sum_{k=1}^{n} \zeta_{k}^{2}
$$

where $\zeta_{k}$ is the mean error in the proper motion of the kth star. The error in $\sigma_{i}^{2}$ consists of two parts; the error in $\sigma_{o}$ and errors in the determination of $\zeta_{k}$. These errors are given by

$$
\begin{gathered}
\epsilon_{1}=\sigma_{o}^{2}\left(\frac{2}{n}\right)^{\frac{1}{2}} \\
\epsilon_{2}=\frac{2^{1 / 2}}{n}\left(\sum_{k=1}^{n} \frac{\zeta_{k}^{4}}{n_{k}}\right)^{1 / 2},
\end{gathered}
$$

where $n_{k}$ is the number of observations for star $k$. The total error in $\sigma_{i}^{2}$ is then

$$
\epsilon_{i}=\left(\epsilon_{1}^{2}+\epsilon_{2}^{2}\right)^{1 / 2} .
$$

As noted earlier, the errors in the motions derived from the FGS are highly non-uniform. However, the above formalism assumes that all the stars are taken from a single parent population. To address this issue only stars that had 55 or more observations were used to compute the proper motion dispersions. Cluster members were defined as the subset of stars whose proper

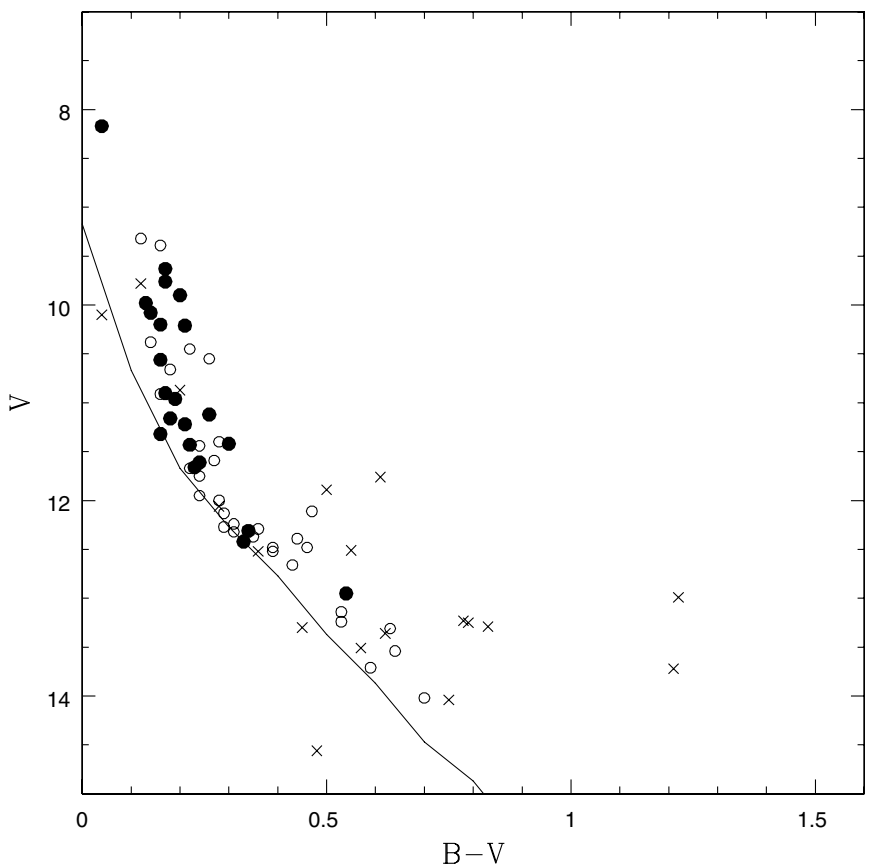

Figure 4. Distribution of our sample M35 stars in the cluster's color-magnitude diagram. Solid circles represent stars used to compute the cluster's proper motion dispersions. Open circles represent stars that did not have more than 55 observations, had anomalous motions, or larger than expected proper motion errors. Crosses represent non members. The accuracy of the internal motion dispersions could be improved if additional observations of the open circle stars were obtained.

motions in both right ascension and declination were less than $1.5 \operatorname{arcsec}^{-}$century ${ }^{-1}$ and whose position in the M35 color-magnitude diagram was consistent with membership. Stars 631 and 845 were also dropped from this sample because of their anomalously high motions and errors. This process reduced the sample size from 74 to 22 stars. Only these 22 stars were used in the internal proper motion analysis (see Figure 4). The value of $\sigma_{i}$ computed using this sample was $0.019 \pm 0.003$ arcsec century $^{-1}$ in right ascension and 0.018 \pm 0.003 arcsec century $^{-1}$ in declination. If the proper motion centroids were allowed to differ from zero these values became $0.018 \pm 0.003$ and $0.018 \pm 0.003$ arcsec century $^{-1}$, respectively. The average one-coordinate internal proper motion dispersion is, therefore, $0.018 \pm 0.002 \operatorname{arcsec}$ century ${ }^{-1}$.

\section{CLUSTER DISTANCE AND AGE}

If the motions within M35 are isotropic, it is possible to estimate the cluster's distance by equating its proper motion 


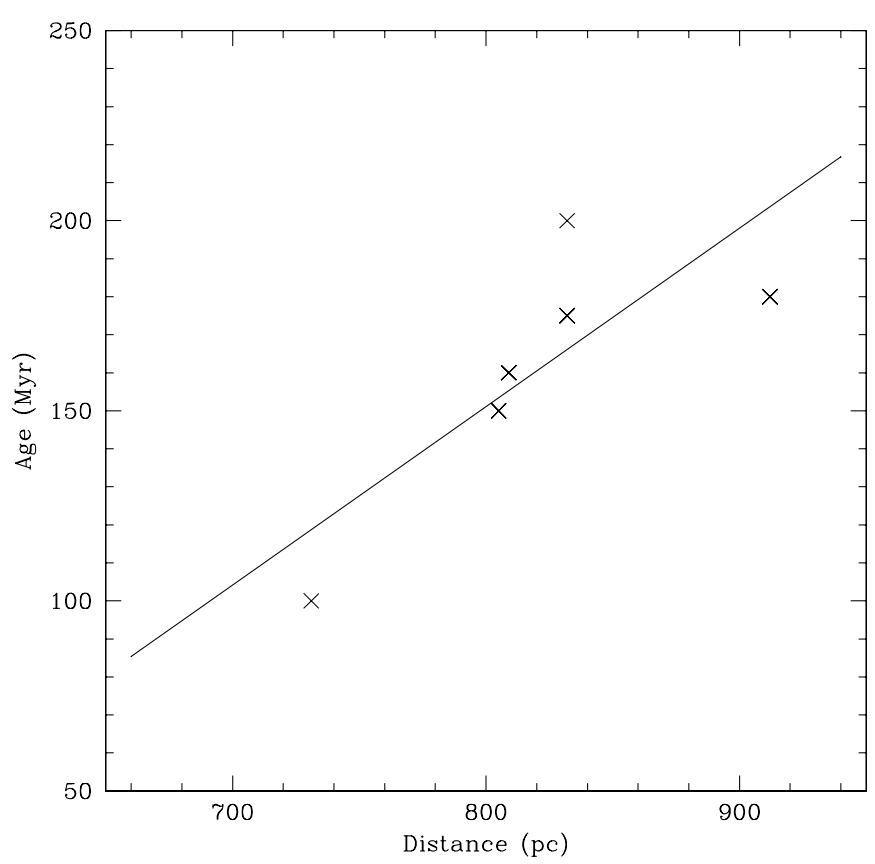

Figure 5. Roughly linear relation exists between the estimated values of the distance and age of M35. This figure shows that relation based on the values provided in Kalirai et al. (2003). The dynamical M35 distance of 762 pc implies an age of about 133 Myr.

and radial velocity dispersions. In this case, the assumption of isotrophy is justified by the fact that the measured proper motion dispersions in right ascension and declination are, to within their errors, the same. Geller et al. (2010) obtained 5201 radial velocity measurements of 1144 stars in M35 as part of their ongoing study of that object. These stars fell within the magnitude range $13.0 \leqslant V \leqslant 16.5$ which for M35 corresponds to a mass range of between 1.6 and 0.8 solar masses. They found an upper limit to the cluster's radial velocity dispersion of $0.81 \pm$ $0.08 \mathrm{~km} \mathrm{~s}^{-1}$. These investigators did not report any variation in this dispersion with magnitude. Therefore, it is used in the below analysis even though the magnitude ranges of the proper motion and radial velocity stars are not identical. After making a correction for undetected binaries, they estimate that the true radial velocity dispersion is $0.65 \pm 0.10 \mathrm{~km} \mathrm{~s}^{-1}$. Equating this value to the prior proper motion dispersion of $0.018 \pm$ 0.002 arcsec century $^{-1}$ gives a cluster distance of $762 \pm 145 \mathrm{pc}$. This is comparable to the value found by other investigators who employed photoelectric or CCD measurements and cluster main-sequence fitting techniques. Those values vary from 725 to $912 \mathrm{pc}$. A detailed discussion of these distance determinations can be found in Kalirai et al. (2003).

The primary quantity responsible for the large range in M35 distance estimates obtained from main-sequence fitting is the assumed cluster age. A roughly direct linear relation exists between the cluster's distance and age. Smaller distances are found when a low cluster age is used and vice versa. These values are coupled through the use of cluster isochrones. M35 ages and distances determined since 1999 are provided in Table 1 of Kalirai et al. (2003). A plot of these values is shown in Figure 5. Based on this figure and the dynamical cluster distance of $762 \mathrm{pc}$, M35 has an age of about 133 Myr. Although this value is within the range commonly associated with M35, we caution that it is based on the crude relation shown in Figure 5. Even if this relation is accepted, the relatively large error in the dynamical distance allows ages extending from $65 \mathrm{Myr}$ to 201 Myr.

\section{SUMMARY}

We have used one-hundred and eight orbits of FGS data, spanning 14 years, to compute the relative proper motions of 74 stars in M35. Based on the proper motions of 22 of these stars that were measured with high precision and whose membership was confirmed by their location in the cluster's color-magnitude diagram, we find that the M35 one-dimensional proper motion dispersion is $0.018 \pm 0.002$ arcsec century $^{-1}$. When combined with the M35 binary corrected radial velocity dispersion measurement provided by Geller et al. (2010) of $0.65 \pm 0.10 \mathrm{~km} \mathrm{~s}^{-1}$, a dynamical distance of $732 \pm 145$ pc results. The largest source of error in this estimate is caused by the uncertainty in the radial velocity dispersion. Unfortunately, decreasing the size of this error will be challenging task since thousands of additional radial velocity measurements would be required. Therefore, it is unlikely that this distance estimate can be substantially improved in the near future. Based on this newly derived dynamical distance, M35 has an age of about 133 Myr.

\section{REFERENCES}

Allen, C. 1976, Astrophysical Quantities (London: The Athlone Press) Benedict, G., et al. 1992, PASP, 104, 958

Cudworth, K. 1971, AJ, 76, 475

Cudworth, K. 1976a, AJ, 81, 519

Cudworth, K. 1976b, AJ, 81, 975

Cudworth, K. 1979a, AJ, 84, 1312

Cudworth, K. 1979b, AJ, 84, 1866

Ebbighausen, E. 1942, AJ, 50, 1

Geffert, M., Kuemmel, M., \& Schmidt, H. 1995, A\&AS, 112, 229

Geller, A., Mathieu, R., Braden, E., Meibom, S., Platais, I., \& Dolan, C. 2010, AJ, 139, 1383

Hemenway, P., et al. 1997, AJ, 114, 2796

Jones, B. 1970, AJ, 75, 563

Kalirai, J., Fahlman, G., Richer, G., \& Ventura, P. 2003, AJ, 126, 1402

Leonard, P., \& Merritt, D. 1989, ApJ, 339, 195

McNamara, B. 1976, AJ, 81, 375

McNamara, B., Harrison, T., \& Baumgardt, H. 2004, ApJ, 602, 264

McNamara, B., \& Sanders, W. 1977, A\&A, 54, 569

McNamara, B., \& Sanders, W. 1978, A\&A, 62, 259

McNamara, B., \& Sanders, W. 1983, A\&A, 118, 361

McNamara, B., \& Sekiguchi, K. 1986a, AJ, 91, 557

McNamara, B., \& Sekiguchi, K. 1986b, ApJ, 310, 613

Sung, H., \& Bessell, M. 1999, MNRAS, 306, 361

van der Marel, R., \& Anderson, J. 2010, ApJ, 710, 1063 